\title{
Motion of Vibration Manipulators with Self Stopping Device in One Direction with Interactions of Two Non Deformable Elements in One Direction
}

\author{
Kazimieras RAGULSKIS*, Bronislovas SPRUOGIS**, Marijonas BOGDEVIČIUS***, \\ Arvydas MATULIAUSKAS $* * * *$, Vygantas MIŠTINAS $* * * * *$, Liutauras RAGULSKIS******* \\ *Kaunas University of Technology, K. Donelaičio Str. 73, LT-44249, Kaunas, Lithuania, E-mail: kazimieras3@hotmail.com \\ **Vilnius Gediminas Technical University, Plytiness Str. 27, LT-10105, Vilnius, Lithuania, \\ E-mail: bronislovas.spruogis@vgtu.lt \\ ***Vilnius Gediminas Technical University, Plytinès Str. 27, LT-10105, Vilnius, Lithuania, \\ E-mail: marijonas.bogdevicius@vgtu.lt \\ ****Vilnius Gediminas Technical University, Plytinès Str. 27, LT-10105, Vilnius, Lithuania, \\ E-mail: arvydas.matuliauskas@vgtu.lt \\ *****Vilnius Gediminas Technical University, Plytinès Str. 27, LT-10105, Vilnius, Lithuania, \\ E-mail:vygantas.mistinas@vgtu.lt \\ ******Vytautas Magnus University, Vileikos Str. 8, LT-44404, Kaunas, Lithuania, E-mail: l.ragulskis@if.vdu.lt \\ cross $^{\text {ref }}$ http://dx.doi.org/10.5755/j01.mech.26.6.25527
}

\section{Introduction}

A number of principles of operation and structures of vibromotors are created the action of which is based on vibrations and waves together with nonlinear dynamical qualities. Their use is constantly widening in the newly created manipulators, robots because of their precision, dynamicity, from possible small to big dimensions and weights. Here a vibromotor is presented, which moves together with the displaced body according to the one dimensional coordinate and is based on normal interactions of two rigid bodies, which coincide with the direction of motion of the displaced body. The chosen parameters enable to avoid stationary multivalued motions in the vicinities of resonances. Self stopping device is included in the structure of the system, which limits the motion of the displaced body in one direction. The investigations are performed by analytical - numerical methods. The material presented by analytical relationships and graphically determines the qualities of the system and the influence of parameters to the results of operation of the system.

In the field of robots wide and deep investigations are performed by V. A. Glazunov himself and his scientific school [1, 2, 3 and a number of other publications by them]. They created new mechanisms in the field of investigation of mechanics of robots. The problem of investigation of manipulators and robots with vibration drives arises. Vibration driven original robot with an electromagnetic actuator and an opposing spring is investigated in [4]. New methods and means of dynamic synchronization of pneumatic vibroexciters as well as of some other types of autonomic vibroexciters and their investigations performed in the middle of the previous century are presented in publications of some of the authors. New structures were created and results of investigations were developed. Industrial robots for miniature products are intensively developed and are investigated in earlier publications of some of the authors. New structures of precision microrobots with vibromotors were created and the results of investigations were developed in earlier publications of some of the au- thors. Investigations of pipe robots are intensively developed and new structures are created. Here one can note a robot performing motion inside the pipe which is investigated in [5], self-exciting vibration of a pipe robot is investigated in [6], multi function pneumatic changers, characteristics of pneumatic transducers and synchronization of pneumatic vibroexciters are investigated in [7] and other related publications. Further a number of obtained scientific results serve for this purpose: resonances of nonlinear vibrating systems are investigated in [8], periodic orbits in mechanical systems are investigated in [9] and vibroimpact energy sink is investigated in [10].

First schematic representation of the investigated system is presented. Then the model of the system is described. Numerical investigations for various parameters of the system are performed. Amplitude frequency characteristics are obtained. Optimal regime of operation of the investigated system is determined.

The obtained results are used in the process of design of vibration manipulators.

2. Model for the investigation of dynamics of a vibration manipulator (Fig. 1).

The system which has two stages is investigated

Equations of motion of the system when $\dot{x}_{2}>0$ are the following ones:

$$
\begin{aligned}
& m_{1} \ddot{x}_{1}+H\left(\dot{x}_{1}-\dot{x}_{2}\right)+C\left(x_{1}-x_{2}\right)=F(\omega t), \\
& m_{2} \ddot{x}_{2}-H\left(\dot{x}_{1}-\dot{x}_{2}\right)-C\left(x_{1}-x_{2}\right)+H_{0} \dot{x}_{2}=-F(\omega t),
\end{aligned}
$$

where: $\omega$ is the radial frequency of the external exciting force.

duced:

In the Eq. (1) the following notations are intro-

$$
\mu=\frac{m_{2}}{m_{1}}, \tau=p t, p=\sqrt{\frac{C}{m_{1}}}, d \tau=p d t, \dot{x}=p x^{\prime},
$$




$$
\begin{aligned}
& { }^{\prime}=\frac{d}{d \tau}, \cdot=\frac{d}{d t}, 2 h=\frac{H}{\sqrt{C m_{1}}}, 2 h_{0}=\frac{H_{0}}{\sqrt{C m_{3}}}, \\
& \nu=\frac{\omega}{p}, f(v \tau)=\frac{F(v \tau)}{C} .
\end{aligned}
$$

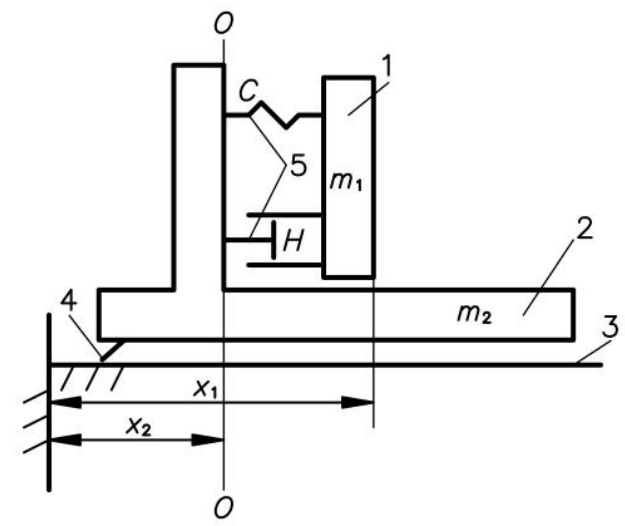

a) Schematic representation of the vibration manipulator

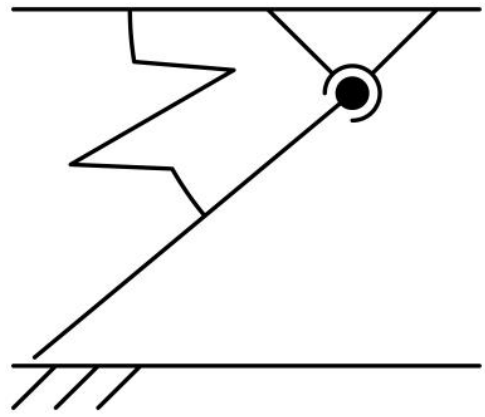

b) Self stopping device

Fig. 1 Model of the system: $m_{1}$ and $m_{2}$ are masses of the input and output elements; $x_{1}$ and $x_{2}$ are the coordinates of the interacting surfaces of the input and output interacting elements; 3 is an immovable surface; 4 is a self stopping device $\dot{x}_{2}<0 ; 5$ are connecting elements with the coefficient of stiffness $C$ and the coefficient of viscous damping $H ; 0-0$ is the static position of equilibrium of the interacting surface of element 2 with respect to element 2

The Eq. (1) respectively become the following

ones:

$$
\begin{aligned}
& x_{1}^{\prime \prime}+2 h\left(x_{1}^{\prime}-x_{2}^{\prime}\right)+x_{1}-x_{2}=f(v \tau), \\
& \mu x_{2}^{\prime \prime}-2 h\left(x_{1}^{\prime}-x_{2}^{\prime}\right)+2 h_{0} x_{2}^{\prime}-x_{1}+x_{2}=-f(v \tau) .
\end{aligned}
$$

Assuming harmonic excitation:

$$
\begin{aligned}
& x_{1}^{\prime \prime}+2 h\left(x_{1}^{\prime}-x_{2}^{\prime}\right)+x_{1}-x_{2}=f \sin (v \tau), \\
& \mu x_{2}^{\prime \prime}-2 h\left(x_{1}^{\prime}-x_{2}^{\prime}\right)+2 h_{0} x_{2}^{\prime}-x_{1}+x_{2}=-f \sin (v \tau) .
\end{aligned}
$$

The following value of the force is calculated:

$$
P=-f \sin (v \tau)+2 h\left(x_{1}^{\prime}-x_{2}^{\prime}\right)-2 h_{0} x_{2}^{\prime}+x_{1}-x_{2} .
$$

Calculation according to the presented equations enables to investigate dynamical qualities of the analysed system.

\section{Results of investigation of forced vibrations of the vibration manipulator}

In this case investigation is performed by assuming:

$$
\mu=4, h=0.1, h_{0}=0.1, f=1 .
$$

Initial non dimensional displacements and velocities are assumed equal to zero.

Two periods of steady state motion are investigated.

3.1. Results of investigation of dynamics of the system before the resonance

The following value of non dimensional frequency of excitation is investigated:

$$
v=0.5 \text {. }
$$

Amplitude frequency characteristics (constant part and first three harmonics) of steady state regime are shown in Fig. 2.

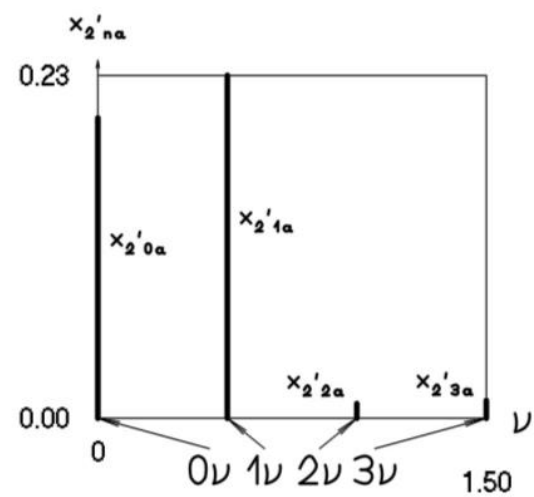

Fig. 2 Amplitude frequency characteristics (constant part and first three harmonics) of steady state regime of a vibration manipulator before the resonance

Dynamics of the system is presented in Fig. 3 . Non dimensional displacement of the first degree of freedom as function of non dimensional time, non dimensional displacement of the second degree of freedom as function of non dimensional time, non dimensional velocity of the first degree of freedom as function of non dimensional time, non dimensional velocity of the second degree of freedom as function of non dimensional time, difference of non dimensional displacements as function of non dimensional time, force as function of non dimensional time, difference of non dimensional displacements as function of the second non dimensional displacement are presented.

From the obtained results it is determined that the constant part and the first harmonic are much larger than further harmonics.

3.2. Results of investigation of dynamics of the system in the vicinity of resonance

The following value of non dimensional frequency of excitation is investigated:

$$
v=1 .
$$


Dynamics of the system is presented in Fig. 4. Non dimensional displacement of the first degree of freedom as function of non dimensional time, non dimensional displacement of the second degree of freedom as function of non dimensional time, non dimensional velocity of the first degree of freedom as function of non dimensional time, non dimensional velocity of the second degree of freedom as function of non dimensional time, difference of non dimensional displacements as function of non dimen- sional time, force as function of non dimensional time, difference of non dimensional displacements as function of the second non dimensional displacement are presented.

Amplitude frequency characteristics (constant part and first three harmonics) of steady state regime are shown in Fig. 5.

From the obtained results it is determined that the constant part and the first harmonic are much larger than further harmonics.

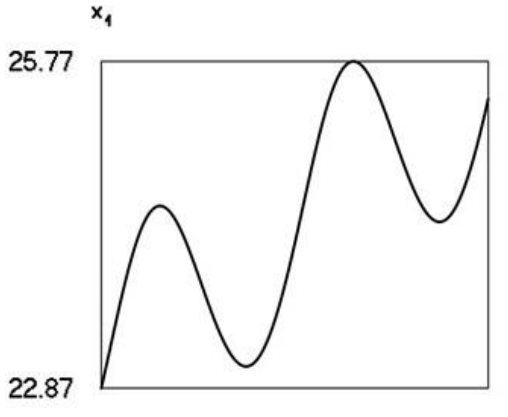

226.19
251.33

$\tau \quad 23.07$

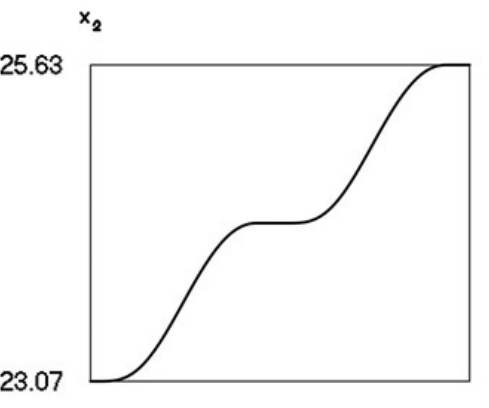

226.19

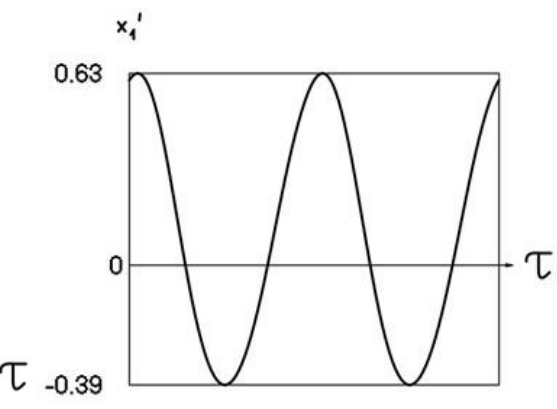

251.33
226.19 a

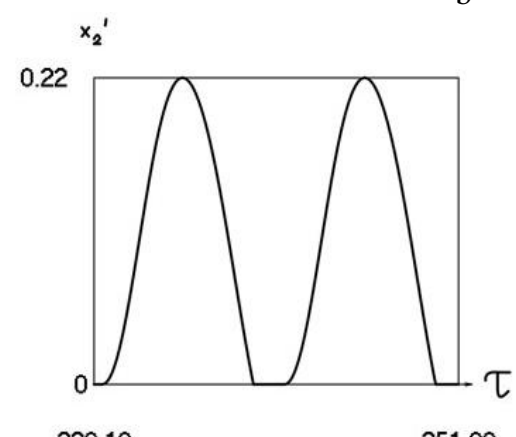

P

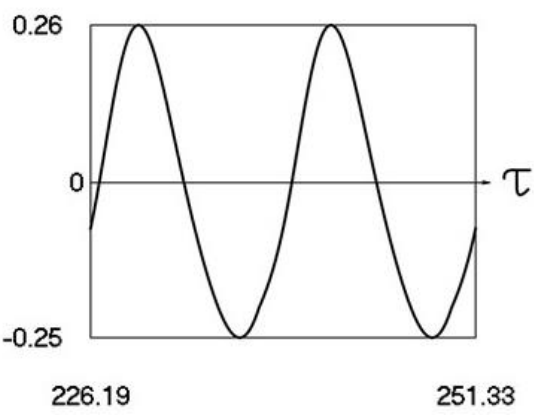

$\mathrm{f}$

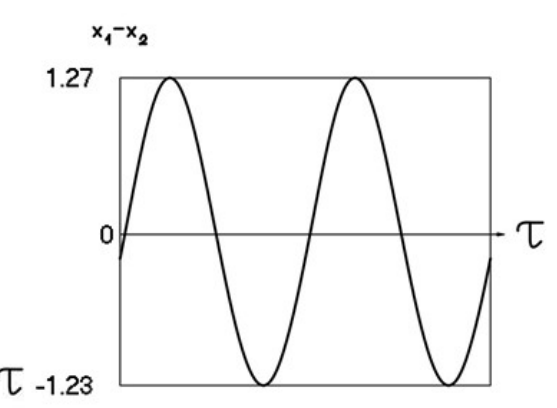

226.19

251.33

e

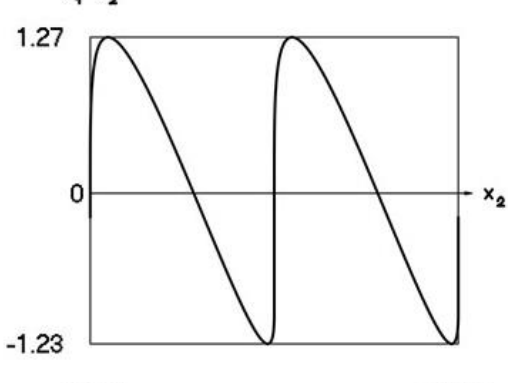

g

Fig. 2 Dynamics of the system before the resonance

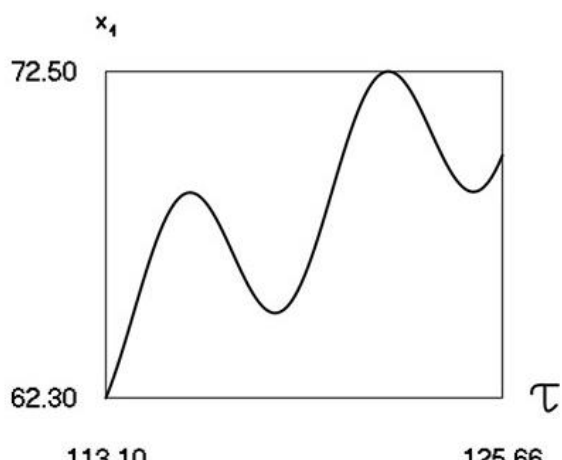

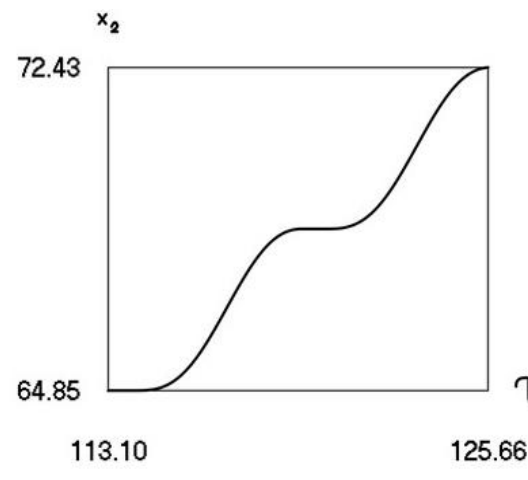

b

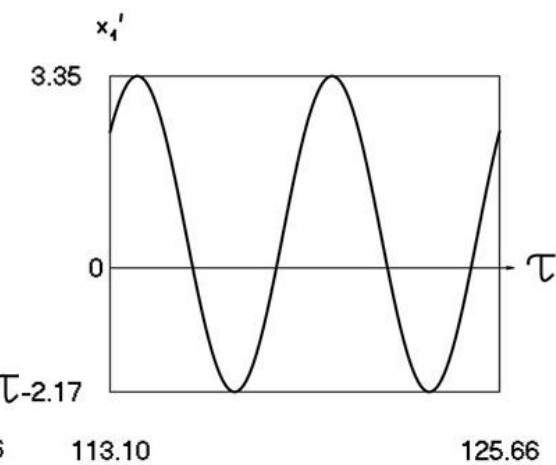

c

Fig. 4 Dynamics of the system in the vicinity of resonance 


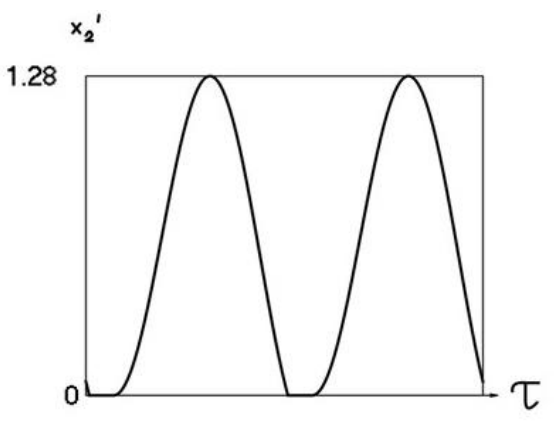

113.10

d

$P$

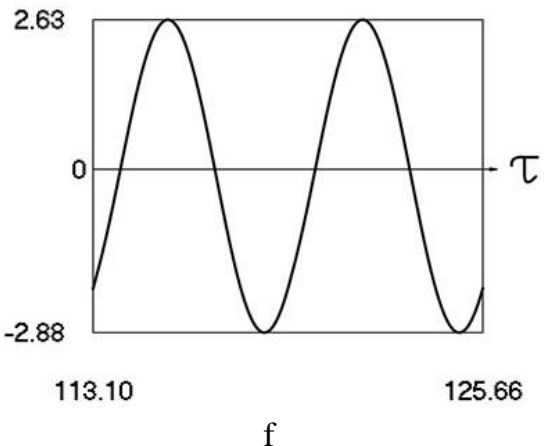

113.10 125.66

125.66

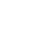

Fig. 4 Continuation

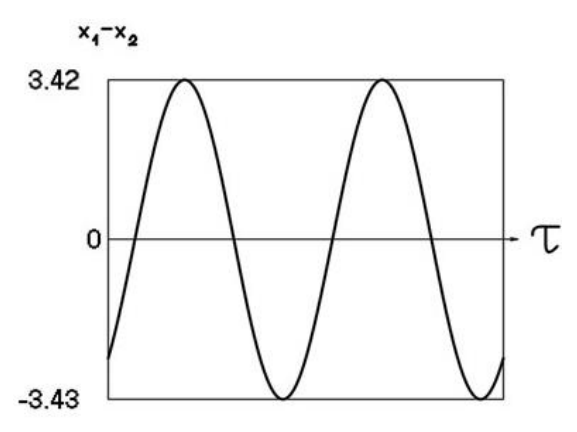

113.10

125.66

e

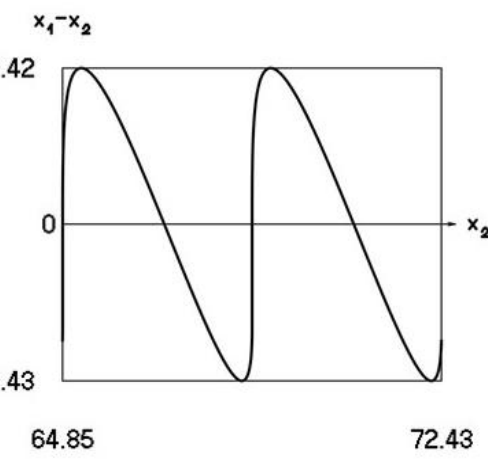

g

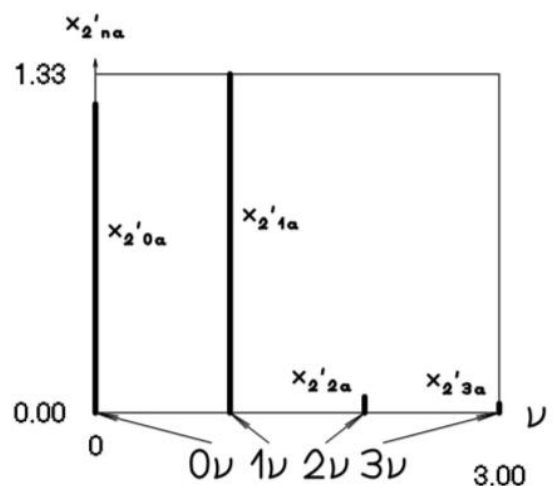

Fig. 5 Amplitude frequency characteristics (constant part and first three harmonics) of steady state regime of a vibration manipulator in the vicinity of resonance

3.3. Results of investigation of dynamics of the system after the resonance

The following value of non dimensional frequency of excitation is investigated:

$$
v=2 .
$$

Dynamics of the system is presented in Fig. 6. Non dimensional displacement of the first degree of freedom as function of non dimensional time, non dimensional displacement of the second degree of freedom as function of non dimensional time, non dimensional velocity of the first degree of freedom as function of non dimensional time, non dimensional velocity of the second degree of freedom as function of non dimensional time, difference of non dimensional displacements as function of non dimensional time, force as function of non dimensional time, difference of non dimensional displacements as function of the second non dimensional displacement are presented.

Amplitude frequency characteristics (constant part and first three harmonics) of steady state regime are shown in Fig. 7.

From the obtained results it is determined that the constant part and the first harmonic are much larger than further harmonics.

\subsection{Conclusions about the results of investigation of dy-} namics of the system

The presented graphical relationships reveal the dynamic behavior of the investigated system for various parameters of the analysed dynamical model of a vibration manipulator. Some of the graphical relationships for the three investigated cases (before the resonance, in the vicinity of the resonance and after the resonance) look similar to each other, but one is to take into account substantial differences in the values of the represented quantities. From the obtained results it is determined that the constant part and the first harmonic are much larger than further harmonics and that their values substantially depend on the frequency of excitation.

From the presented results it can be noted that the average velocity of motion of the investigated system in the vicinity of resonance is much higher than before the resonance and than after the resonance. Thus the problem of determination of optimal frequency of excitation arises and it is solved further in this paper. 

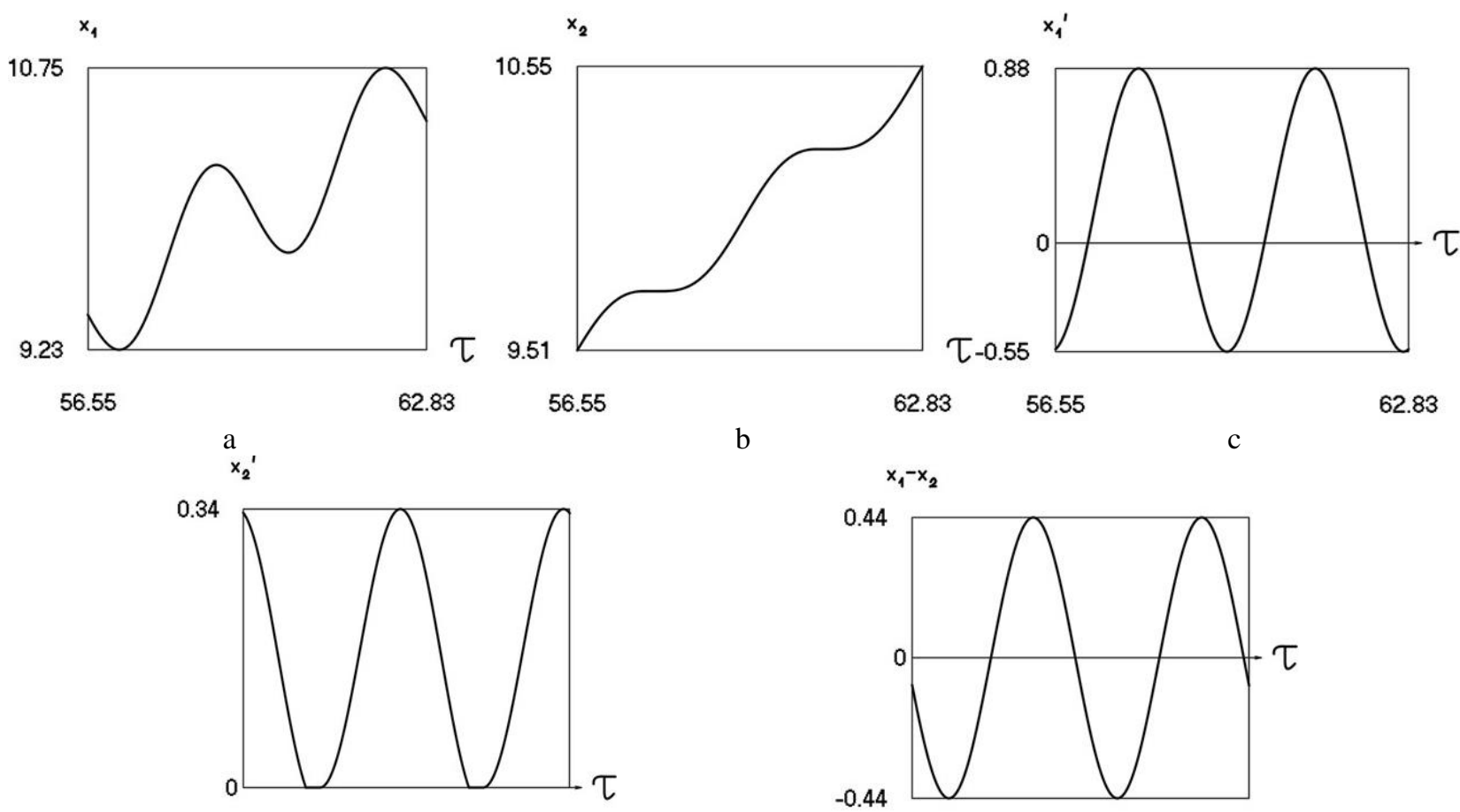

b

c
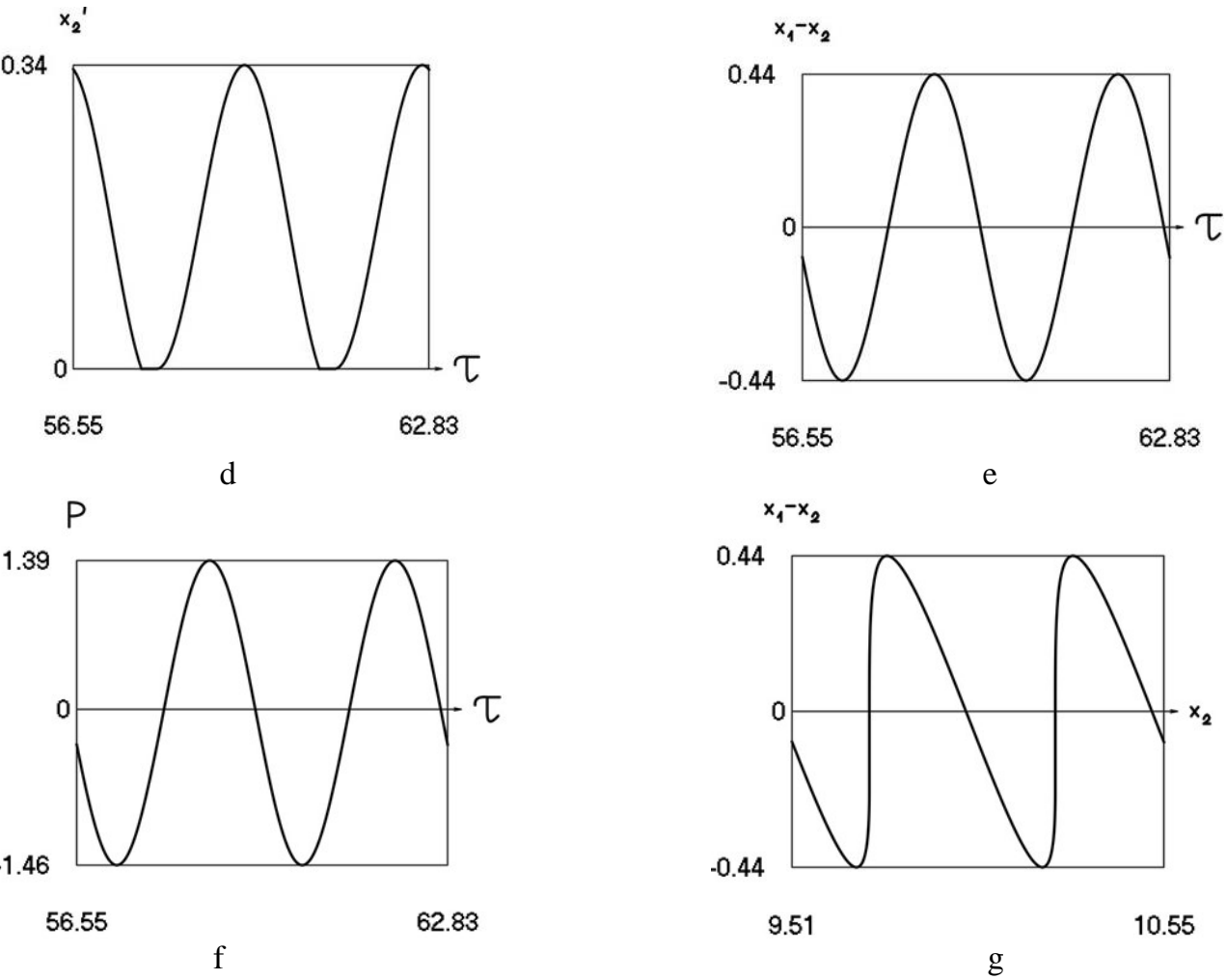

Fig. 6 Dynamics of the system after the resonance

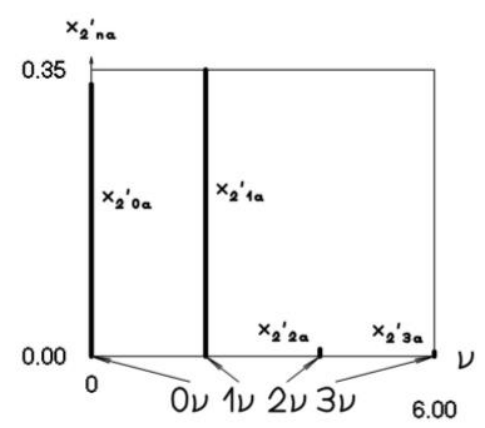

Fig. 7 Amplitude frequency characteristics (constant part and first three harmonics) of steady state regime of a vibration manipulator after the resonance

\section{Determination of optimal operation regime of the vibration manipulator}

Average velocity of the second degree of freedom in steady state regime as function of frequency of excitation for various values of amplitude of excitation is presented in Fig. 8. Average velocity as function of frequency of excitation corresponds with the results presented in the amplitude frequency characteristics.

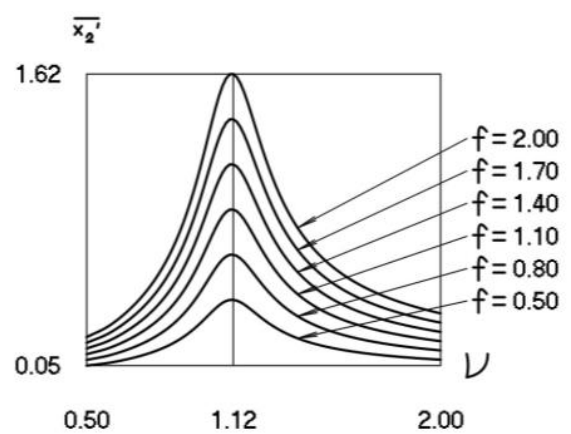

Fig. 8 Average velocity of the second degree of freedom in steady state regime as function of frequency of excitation for various values of amplitude of excitation of a vibration manipulator

From the obtained results optimal frequency of excitation corresponding to maximum average velocity of the second degree of freedom in steady state regime is determined and is indicated in the figure. From the obtained results it is seen that optimal frequency of excitation is shifted from the resonance frequency investigated earlier. This fact takes place because of damping and nonlinear effects in the investigated vibration manipulator. 


\section{Conclusions}

The investigated vibration manipulator is a nonlinear dynamical system. Here two qualities are used, namely the direction of the force of vibroexciter coincides with the direction of motion of the manipulator and the choice of the frequency of the vibroexciter is performed by taking into account the eigenfrequency of the system.

In steady state regimes of motion of the system graphical relationships representing motions, forces, power and frequencies were obtained before, after and at resonance representing dynamical behaviour of the system. From the obtained results it is determined that the constant part and the first harmonic are much larger than further harmonics and that their values substantially depend on the frequency of excitation.

The presented graphical relationships reveal the dynamic behavior of the investigated system for various parameters of the analysed dynamical model of a vibration manipulator. From the presented results it can be noted that the average velocity of motion of the investigated system in the vicinity of resonance is much higher than before the resonance and than after the resonance. Thus the problem of determination of optimal frequency of excitation arises and it is solved in this paper.

From the obtained results optimal frequency of excitation corresponding to maximum average velocity of the second degree of freedom in steady state regime is determined. From the obtained results it is seen that optimal frequency of excitation is shifted from the resonance frequency investigated earlier. This fact takes place because of damping and nonlinear effects in the investigated vibration manipulator.

The obtained graphical relationships show that the operation of the system is effective according to the maximum value of velocity of the system and minimum losses of dissipative forces. Those properties are used for the case of resonance when the frequency of the vibroexciter coincides with the eigenfrequency of the system.

Results of investigations enable to create multidimensional complicated robots which effectively operate in steady state regimes.

\section{References}

1. Glazunov, V. 2018. New Mechanisms in Contemporary Robot Engineering. Moscow: Tehnosphere, (in Russian).

2. Pavlovsky, V. 2013. For elaboration of walking machines, Keldysh Institute Preprints 101: 1-32. (in Russian).

3. Glazunov, V.; Heylo, S.; Kostiukov, A. 2016. Investigation of vibrations of a mechanism with parallel structure, vibration technologies, Mechatronics and Controlled Machines, p. 23-28. (in Russian).

4. Bolotnik, N. N.; Nunuparov, A. M.; Chashchukhin, V. G. 2016. Capsule-type vibration-driven robot with an electromagnetic actuator and an opposing spring: dynamics and control of motion, Journal of Computer and Systems Sciences International 55(6): 986-1000.
https://doi.org/10.1134/S106423071605004X.

5. Spruogis, B.; Ragulskis, K.; Bogdevičius, M.; Ragulskis, M.; Matuliauskas, A.; Mištinas, V. 2002. Robot Performing Stepping Motion Inside the Pipe. Patent LT 4968 B.

6. Ragulskis, K.; Bogdevičius, M.; Mištinas, V. 2008. Behaviour of dynamic processes in self-exciting vibration of a pipe robot, Journal of Vibroengineering 10(3): 397-399.

7. Kibirkštis, E.; Pauliukaitis, D.; Miliūnas, V.; Ragulskis, K. 2017. Synchronization of pneumatic vibroexciters under air cushion operating mode in a selfexciting autovibration regime, Journal of Mechanical Science and Technology 31(9): 4137-4144. https://doi.org/10.1007/s12206-017-0809-6.

8. Wedig, W. V. 2016. New resonances and velocity jumps in nonlinear road-vehicle dynamics, Procedia IUTAM 19: 209-218. https://doi.org/10.1016/j.piutam.2016.03.027.

9. Spedicato, S.; Notarstefano, G. 2017. An optimal control approach to the design of periodic orbits for mechanical systems with impacts, Nonlinear Analysis: Hybrid Systems 23: 111-121.

https://doi.org/10.1016/j.nahs.2016.08.009.

10. Li, W.; Wierschem, N. E.; Li, X.; Yang, T. 2018. On the energy transfer mechanism of the single-sided vibro-impact nonlinear energy sink, Journal of Sound and Vibration 437: 166-179. https://doi.org/10.1016/j.jsv.2018.08.057.

K. Ragulskis, B. Spruogis, M. Bogdevičius, A. Matuliauskas, V. Mištinas, L. Ragulskis

\section{MOTION OF VIBRATION MANIPULATORS WITH SELF STOPPING DEVICE IN ONE DIRECTION WITH INTERACTIONS OF TWO NON DEFORMABLE ELE- MENTS IN ONE DIRECTION}

S u m m a r y

Vibration manipulators are important in the process of creation of 1D - 3D manipulators and robots. The manipulator which is proposed here has the following advantages: simplicity of the structure and high coefficient of useful operation. This is because of the fact that the driving force provided by the vibration drive coincides with the direction of motion of the manipulator. Analysis of the regime of steady state motion is presented by using numerical methods. The obtained graphical relationships enable to choose the most effective parameters of the system in the process of design of vibration manipulators.

Keywords: vibration manipulator, 1D, direction of the driving force, direction of motion, resonance regime.

Received March 18, 2020

Accepted December 01, 2020 